\title{
Systemic lupus erythematosus presenting with cardiac tamponade due to a haemorrhagic pericardial effusion
}

\author{
T. Rudra, P.A. Evans and E.N. O'Brien \\ Department of Medicine, Enfield District Hospital, Southgate, London N21, UK.
}

\begin{abstract}
Summary: A 14 year old girl presented with cardiac tamponade due to a haemorrhagic pericardial effusion. Systemic lupus erythematosus was diagnosed. Pericardial stripping was performed due to recurrence of the effusion despite pericardiocentesis and steroid therapy.
\end{abstract}

\section{Introduction}

Pericarditis is the commonest cardiac complication of systemic lupus erythematosus (SLE). ${ }^{1}$ However, pericardial effusion causing cardiac tamponade is rare in $\mathrm{SLE}^{2}$ and it is even rarer for it to be the presenting feature of SLE. ${ }^{3}$ We present a case of SLE presenting with cardiac tamponade due to a haemorrhagic pericardial effusion.

\section{Case report}

The patient was a 14 year old girl of Turkish origin. She had been apparently well until 2 months before admission when she had transient arthralgia of her hands, elbows and knees. She subsequently developed a 'flu-like illness' which was associated with a low grade fever and a persistent non-productive cough. Three days before admission she became more breathless and was referred to us for further investigation.

On examination she was of thin build with pale conjuctivae. Her temperature on admission was $38^{\circ} \mathrm{C}$. There were palpable lymph nodes in the neck, right axilla and both inguinal regions. She was not dyspnoeic at rest. The pulse was $120 /$ minute, blood pressure $120 / 70 \mathrm{~mm} \mathrm{Hg}$, with no paradoxical pulse. Jugular venous pressure was elevated. Examination of her chest revealed signs of bilateral pleural effusions and an audible pericardial rub.

Other investigations at this stage revealed a haemoglobin concentration of $8.6 \mathrm{~g} / \mathrm{dl}$, white cell count $11.4 \times 10^{9} / 1$, ESR $64 \mathrm{~mm} / \mathrm{h}$. Blood urea, electrolytes and liver and thyroid function tests were

Correspondence: T. Rudra, M.R.C.P., Bridgend General Hospital, Bridgend, South Wales, UK.

Accepted: 20 January 1987 normal. There was microscopic haematuria and proteinuria; blood cultures proved sterile.

A diagnostic pericardial aspirate revealed a haemorrhagic effusion with a protein content of $49 \mathrm{~g} / \mathrm{l}$, polymorphs and mononuclear cells. Staining for alcohol and acid-fast bacilli was negative and culture revealed no growth.

A diagnostic pleural aspiration revealed straw coloured fluid with a protein content of $21 \mathrm{~g} / \mathrm{l}$, and the presence of polymorphs and lymphocytes. Culture proved negative as was the stain for alcohol and acidfast bacilli.

Over the next few days she became more breathless and developed orthopnoea. The pulse rate was $150 /$ minute and the blood pressure $100 / 70 \mathrm{~mm} \mathrm{Hg}$ with a paradoxical pulse of $20 \mathrm{~mm} \mathrm{Hg}$. Chest X-ray showed an enlarging cardiac shadow and an echocardiogram confirmed the presence of large pericardial effusions anteriorly and posteriorly. The pericardium was not thickened. A therapeutic pericardiocentesis was performed and $500 \mathrm{ml}$ of blood-stained fluid was aspirated.

At this stage the following results became available: anti-nuclear antibodies - positive (greater than 1:40), DNA binding $71 \%$ (normal 1-10\%). Complement $\mathrm{C}_{3}$ normal, $C_{4}$ decreased at 9 . Tests for the following antibodies were all negative: rheumatoid factor (sheep cell agglutination), antimitochondrial, smooth cell and gastric parietal cell. Thyroid antibodies - positive 1:400 for microsomal antibodies but negative for thyroglobulin. Viral screen - negative.

A diagnosis of systemic lupus erythematosus was made and treatment commenced with prednisolone $40 \mathrm{mg} /$ day. Despite this her condition deteriorated with evidence of re-accumulation of the pericardial effusion. It was therefore decided to excise the pericardium. The anterior pericardium was excised and the fluid aspirated. Two pericardial drains and a pleural 
drain on each side were inserted. She made a good recovery post-operatively. A post-operative echocardiogram showed no re-accumulation of her pericardial effusion.. Histology of the pericardium was that of an organizing fibrinous pericarditis with no evidence of granuloma formation. She made an uneventful recovery. A year later she remained asymptomatic from her SLE but had some cushingoid features.

\section{Discussion}

The most common cardiac manifestation of SLE is pericarditis. The incidence is reported as between $20-$ $30 \% .{ }^{4} \mathrm{However}$ pericarditis is the presenting feature in only $1-4 \%$ of patients with SLE. ${ }^{4}$ Dubois reported no cases of tamponade in 159 cases of lupus pericarditis. ${ }^{4}$ Estes \& Christian in a prospective study of 150 patients with SLE found 29 patients with pericarditis of whom two developed cardiac tamponade. ${ }^{5}$

In SLE the pericardial effusion is exudative in nature. It is usually clear or may be serosanguinous but is very rarely haemorrhagic. On microscopy,

\section{References}

1. Chang, R.W. Cardiac manifestations of SLE. Clin Rheum Dis 1982, 8: 197-205.

2. Dubois, E.L. \& Tuffanellie, D.L. Clinical manifestations of systemic lupus erythematosus. JAMA 1964, 190: 104111.

3. Askerie, A.D. Pericardial tamponade with haemorrhagic fluid in systemic lupus erythematosus. J Am Med Wom Ass 1978, 33: 111-114.

4. Dubois, E.L. A review of the current status of discoid and polymorphonuclear leucocytes are most numerous and monocytes too may be present. Examination of $\cong$ the centrifuged specimen may reveal LE cells which are then diagnostic of SLE. ${ }^{3}$ The pericardial fluid may also demonstrate anti-nuclear and anti-DNA antibodies. ${ }^{6}$

In the absence of cardiac tamponade, lupus pericarditis may be treated with non-steroidal anti-inflam-흐 matory drugs. However, corticosteroids are more $\frac{\bar{m}}{\bar{D}}$ effective in resolving symptoms and eliminating the $\mathbb{\varnothing}$ pericardial effusion., Should cardiac tamponade occur this needs to be drained urgently.

Our patient with SLE developed cardiac tamponade $\vec{\circ}$ and the pericardial fluid was haemorrhagic in nature, $\overrightarrow{\vec{\omega}}$ both of which are rare features of lupus pericarditis. $\stackrel{\omega}{\omega}$ Despite pericardiocentesis and steroid therapy there $\overrightarrow{8}$ was re-accumulation of pericardial fluid resulting in 3 cardiac tamponade which led to surgical excision of os her pericardium. Pericardial stripping or excision is not normally required to prevent recurrence of $\stackrel{A}{A}$ pericardial effusions in SLE once steroid therapy is initiated.

systemic lupus erythematosus and their variants. Unive sity of Southern California Press 1974, pp. 265-275, 40 404.

5. Estes, D. \& Christian, C.L. The natural history of systemic lupus erythematosus by prospective analysis.

6. Goldenburg, D.L., Leff, G. \& Grayzel, A.I. Pericardial tamponade in systemic lupus erythematosus. $N Y$ State $J$ Med 1975, 75: 910-912. 